\title{
Aplicação de Algoritmos Genéticos para o Projeto Ótimo de Manipuladores 3R Considerando Envoltórias Regulares
}

F.R. GONZALEZ ${ }^{1}$, S.F.P. SARAMAGO2${ }^{2}$, Universidade Federal de Uberlândia, Campus Santa Mônica, Av. João Naves de Ávila 2160, Santa Mônica, Uberlândia, MG, Brasil

P.R. BERGAMASCHI ${ }^{3}$, Universidade Federal de Goiás, Campus de Catalão, Av. Dr. Lamartine Pinto de Avelar 1120, 75701-220 Catalão, GO, Brasil.

\begin{abstract}
Resumo. O objetivo deste trabalho é o projeto ótimo de robôs manipuladores 3R, considerando que estes possuem espaço de trabalho cuja envoltória é regular. A otimização caracteriza-se pela obtenção de um espaço de trabalho com volume máximo, minimizando-se as dimensões do robô. O estudo da envoltória é de fundamental importância para o desenvolvimento do projeto de um manipulador, pois representa a fronteira de seu espaço de trabalho. Sua forma depende da arquitetura e das dimensões do robô e tais parâmetros devem ser determinados para que o volume máximo seja obtido. Normalmente, o espaço de trabalho investigado pode apresentar regiões vazias e sua envoltória singularidades que são denominadas pontos de cúspides. Com o surgimento destas singularidades, classifica-se a envoltória como irregular e esta não será considerada durante o processo de otimização. Como método de otimização, utilizou-se algoritmos genéticos. Os autores desenvolveram um código computacional para o cálculo do modelo matemático. Alguns exemplos numéricos são apresentados para verificação e validação da metodologia proposta.
\end{abstract}

\section{Introdução}

Manipuladores 3R são definidos como sendo robôs compostos por três juntas rotacionais, estando o efetuador posicionado na última junta do mesmo. Muito utilizados nas indústrias, estes robôs são objeto freqüente de estudo para pesquisadores da área, sendo que o seu espaço de trabalho é um fator importante na obtenção dos parâmetros ótimos de projeto.

Segundo Kumar e Waldron [8], o espaço de trabalho é uma região definida pelo conjunto de pontos do espaço possíveis de serem alcançados pelo efetuador. No caso de manipuladores 3R, é representado por um sólido de revolução. Roth [11] desenvolveu um dos primeiros trabalhos relacionados com o estudo do espaço de trabalho

\footnotetext{
${ }^{1}$ f.gonzalez@bol.com.br, Faculdade de Engenharia Mecânica, UFU.

${ }^{2}$ saramago@ufu.br, Faculdade de Matemática, UFU.

${ }^{3}$ paulorb@catalao.ufg.br, paulob@innet.psi.br
} 
dos manipuladores. Uma formulação analítica para a determinação da envoltória para estes manipuladores foi proposta por Ceccarelli em 1996 [5]. Abdel-Malek et al [1] desenvolveram um trabalho sobre a determinação de vazios em manipuladores. Wenger [13] mostrou a possibilidade de um manipulador executar movimentos não singulares. Também foram feitos estudos sobre a relação entre as equações do manipulador e o surgimento de regiões de singularidade. Para melhor entendimento sobre o assunto, recomenda-se os trabalhos de Lanni et al [9] e Saramago et al [12].

Neste trabalho, o projeto ótimo de manipuladores 3R é definido como a obtenção do volume de trabalho máximo, mantendo as dimensões do robô dentro de uma faixa limitada, o que significa redução dos custos de fabricação do equipamento e maior faixa de aplicação operacional.

O problema é investigado utilizando algoritmos genéticos, através do código computacional GAOT (Genetic Algorithms Optimization Toolbox). Este programa foi desenvolvido pela Universidade da Carolina do Norte, EUA, conforme Houck et al [6]. A presença de mínimos locais justifica a utilização de métodos randômicos.

No processo de otimização, as variáveis de projeto são os parâmetros do robô (dimensões dos braços e ângulos). O algoritmo escolhe o indivíduo melhor adaptado, ou seja, aquele que gera espaço de trabalho com volume máximo.

Nesta aplicação, o problema de otimização assume como restrição o surgimento de envoltórias irregulares, ou seja, não aceita as envoltórias que apresentam pontos de singularidade (cúspides).

\section{Modelo cinemático de um manipulador 3R}

Um manipulador 3R genérico pode ser esquematizado conforme mostrado na Figura 1.

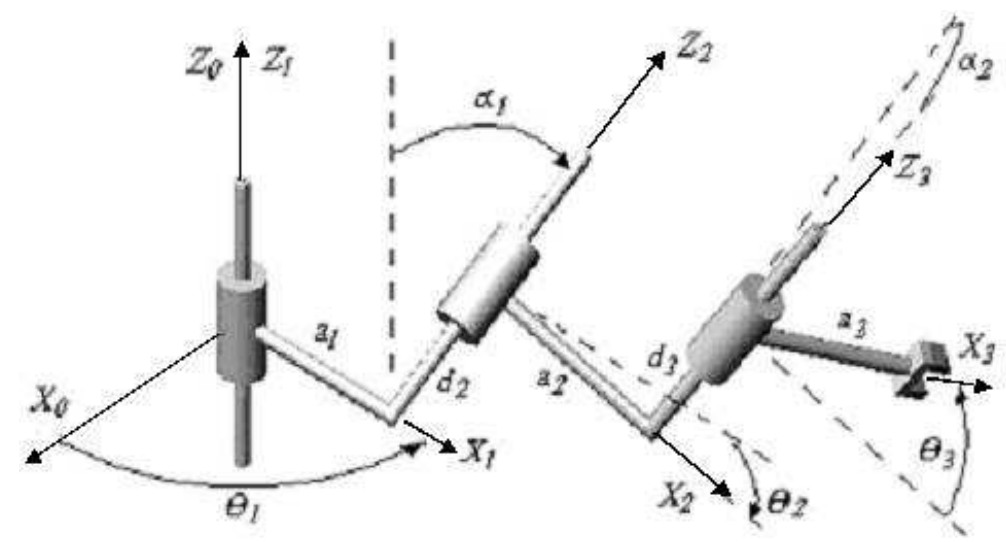

Figura 1: Esquema de um manipulador 3R.

Nesta figura, são visíveis as três juntas rotacionais, as dimensões $a_{1}, d_{2}, a_{2}, d_{3}$ e $a_{3}$ dos braços e os ângulos $\alpha_{1}, \theta_{1}, \alpha_{2}, \theta_{2}$ e $\theta_{3}$. A cada junta do robô, foi associado um sistema de referências que foi utilizado para a determinação das equações 
cinemáticas, a partir da representação de Denavit-Hartenberger. Os ângulos $\theta_{n}$ são variáveis e definem a movimentação do robô. No projeto ótimo, as variáveis de projeto são representadas pelo vetor formado por:

$$
x=\left[\begin{array}{lllllll}
a_{1} & a_{2} & a_{3} & d_{2} & d_{3} & \alpha_{1} & \alpha_{2}
\end{array}\right] .
$$

A partir destes parâmetros, é possível a determinação das equações que representam a posição de um determinado ponto $H$ situado no efetuador do robô em relação ao sistema de base $X_{0} Y_{0} Z_{0}$. A equação (2.1) mostra a matriz de transformação cinemática genérica, entre dois sistemas de coordenadas adjacentes.

$$
T_{i}^{i+1}=\left[\begin{array}{cccc}
c \theta_{i+1} & -s \theta_{i+1} & 0 & a_{i} \\
s \theta_{i+1} c \alpha_{i} & c \theta_{i+1} c \alpha_{i} & s \alpha_{i} & d_{i+1} s \alpha_{i} \\
-s \theta_{i+1} s \alpha_{i} & -c \theta_{i+1} s \alpha_{i} & c \alpha_{i} & d_{i+1} c \alpha_{i} \\
0 & 0 & 0 & 1
\end{array}\right],
$$

sendo $a_{0}=\alpha_{0}=0, d_{1}=0, c \alpha_{i}=\cos \alpha_{i}, s \alpha_{i}=\operatorname{sen} \alpha_{i}, c \theta_{i+1}=\cos \theta_{i+1}$ e $s \theta_{i+1}=$ $\operatorname{sen} \theta_{i+1}, i=0,1,2,3$.

Seja o ponto $H$, definido em relação ao sistema 3 da seguinte forma:

$$
H_{3}=\left[\begin{array}{c}
a_{3} \\
0 \\
0 \\
1
\end{array}\right], \quad \quad\left(a_{3} \neq 0\right)
$$

Para se definir o ponto $H$ em relação ao sistema inercial situado na base do robô, multiplica-se pelas matrizes de transformação [10], resultando em:

$$
H_{0}=\left[T_{0}^{1}\right]\left[T_{1}^{2}\right]\left[T_{2}^{3}\right] H_{3} .
$$

$\mathrm{O}$ vetor que representa $H_{0}$ será então:

$$
\left[\begin{array}{c}
H_{0}^{x} \\
H_{0}^{y} \\
H_{0}^{z} \\
1
\end{array}\right]=\left[\begin{array}{c}
H_{1}^{x} c \theta_{1}-H_{1}^{y} s \theta_{1} \\
H_{1}^{x} s \theta_{1}+H_{1}^{y} c \theta_{1} \\
H_{1}^{z} \\
1
\end{array}\right]
$$

sendo

$$
\begin{aligned}
{\left[\begin{array}{c}
H_{1}^{x} \\
H_{1}^{y} \\
H_{1}^{z} \\
1
\end{array}\right]=} & {\left[\begin{array}{c}
H_{2}^{x} c \theta_{2}-H_{2}^{y} s \theta_{2}+a_{1} \\
H_{2}^{x} s \theta_{2} c \alpha_{1}+H_{2}^{y} c \theta_{2} c \alpha_{1}+H_{2}^{z} s \alpha_{1}+d_{2} s \alpha_{1} \\
-H_{2}^{x} s \theta_{2} s \alpha_{1}-H_{2}^{y} c \theta_{2} s \alpha_{1}+H_{2}^{z} c \alpha_{1}+d_{2} c \alpha_{1} \\
1
\end{array}\right], } \\
& {\left[\begin{array}{c}
H_{2}^{x} \\
H_{2}^{y} \\
H_{2}^{z} \\
1
\end{array}\right]=\left[\begin{array}{c}
a_{3} c \theta_{3}+a_{2} \\
a_{3} s \theta_{3} c \alpha_{2}+d_{3} s \alpha_{2} \\
-a_{3} s \theta_{3} s \alpha_{2}+d_{3} c \alpha_{2} \\
1
\end{array}\right] }
\end{aligned}
$$


Determinando-se $H_{0}$ no espaço cartesiano, é possível a obtenção das coordenadas cilíndricas do mesmo [2]. Estas podem ser definidas por:

$$
\left\{\begin{array}{l}
r^{2}=\left(H_{0}^{x}\right)^{2}+\left(H_{0}^{y}\right)^{2}=\left(H_{1}^{x} c \theta_{1}-H_{1}^{y} s \theta_{1}\right)^{2}+\left(H_{1}^{x} s \theta_{1}+H_{1}^{y} c \theta_{1}\right)^{2} \\
z=H_{0}^{z}=H_{1}^{z}
\end{array}\right.
$$

\section{Volume do espaço de trabalho de um manipu- lador 3R}

A determinação do volume do sólido de revolução que representa o conjunto de posições possíveis para o ponto $H$ necessita da determinação da equação da envoltória. Para a dedução desta equação será necessário parametrizar $r$ e $z$ em função de $\theta_{3}$. Usando as equações (2.2) e (2.3), escreve-se:

$$
r^{2}+z^{2}=\left(H_{2}^{x}\right)^{2}+\left(H_{2}^{y}\right)^{2}+\left(H_{2}^{z}+d_{2}\right)^{2}+2 a_{1}\left(H_{2}^{x} c \theta_{2}-H_{2}^{y} s \theta_{2}\right)+a_{1}^{2} .
$$

Após algumas manipulações algébricas, conforme desenvolvido por Lanni et al [9], obtém-se que:

$$
f\left(r, z, \theta_{3}\right)=\left(r^{2}+z^{2}-A\right)+(C z+D)^{2}+B=0,
$$

sendo

$$
\left\{\begin{array}{l}
A=a_{1}^{2}+a_{2}^{2}+a_{3}^{2}+d_{2}^{2}+d_{3}^{2}+2 a_{2} a_{3} c \theta_{3}+2 d_{2} d_{3} c \alpha_{2}-2 d_{2} a_{3} s \theta_{3} s \alpha_{2} \\
B=-4 a_{1}^{2}\left[\left(a_{3} c \theta_{3}+a_{2}\right)^{2}+\left(a_{3} s \theta_{3} c \alpha_{2}+d_{3} s \alpha_{2}\right)^{2}\right] \\
C=\frac{2 a_{1}}{s \alpha_{1}} \\
D=-\frac{2 a_{1}\left(-a_{3} s \theta_{3} s \alpha_{2}+d_{3} c \alpha_{2}+d_{2}\right) c \alpha_{1}}{s \alpha_{1}}
\end{array}\right.
$$

A envoltória da família de curvas planas de 1-parâmetro (o parâmetro $\theta_{3}$ ) pode ser obtida, segundo Bruce and Giblin [4], como o conjunto de pontos $(r, z)$ que satisfazem às equações:

$$
\left\{\begin{array}{l}
f\left(r, z, \theta_{3}\right)=0 \\
\frac{\partial f\left(r, z, \theta_{3}\right)}{\partial \theta_{3}}=0
\end{array}\right.
$$

Assim, a solução do sistema de equações (3.2) permite que se obtenham as coordenadas cilíndricas da envoltória, as quais são:

$$
\left\{\begin{array}{l}
r=\sqrt{\frac{(C z+D) G+F}{E}+A-z^{2}} \\
z=\frac{-F G \pm \sqrt{-E^{2}\left[B\left(E^{2}+G^{2}\right)+F^{2}\right]}}{C\left(E^{2}+G^{2}\right)}-\frac{D}{C}
\end{array}\right.
$$

sendo

$$
\left\{\begin{array}{l}
E=\frac{\partial A}{\partial \theta_{3}}=-2 a_{2} a_{3} s \theta_{3}-2 d_{2} a_{3} c \theta_{3} s \alpha_{2} \\
F=\frac{1}{2} \frac{\partial B}{\partial \theta_{3}}=4 a_{1}^{2} a_{3}\left[a_{2} s \theta_{3}+a_{3} c \theta_{3} s \theta_{3} s^{2} \alpha_{2}-d_{3} c \theta_{3} s \alpha_{2} c \alpha_{2}\right] \\
G=\frac{\partial G}{\partial \theta_{3}}=\frac{2 a_{1} a_{3} c \theta_{3} s \alpha_{2} c \alpha_{1}}{s \alpha_{1}}
\end{array}\right.
$$


Os parâmetros dados nas equações (3.1) e (3.4) são denominados coeficientes estruturais e dependem das constantes $a_{1}, d_{2}, a_{2}, d_{3}, a_{3}, \alpha_{1}, \alpha_{2}$ e da variável $\theta_{3}$.

As equações (3.3) foram implementadas, através da variação do ângulo $\theta_{3}$, em uma rotina computacional em MATLAB, através da variação do ângulo $\theta_{3}$. A Figura 2-a mostra a seção e a envoltória de um espaço de trabalho, geradas utilizando-se este programa. A Figura 2-b é a representação tridimensional secionada do espaço de trabalho deste robô. Esta mostra também as regiões de vazios e de singularidades (cúspides) que podem surgir com estes tipos de envoltória.

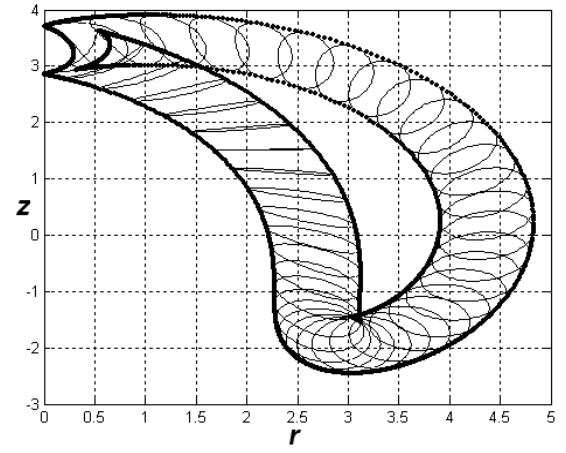

(a)

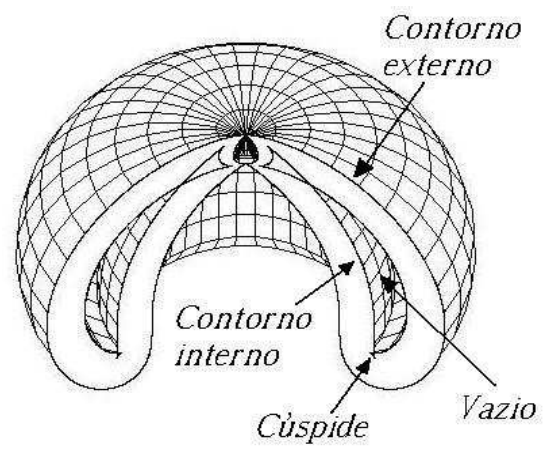

(b)

Figura 2: (a): Seção radial, (b): representação do espaço de trabalho.

O volume do sólido de revolução pode então ser determinado segundo o Teorema de Pappus-Guldin [2], pela expressão:

$$
V=2 \pi r_{G} A,
$$

sendo $A$ a área da seção radial e $r_{G}$ a posição da abscissa do baricentro ou centro geométrico desta seção no plano $(r, z)$. A área da seção é calculada como:

$$
A=A_{\text {ext }}-A_{\text {vazio }}, ; \quad A_{\text {ext }}=\sum_{j=1}^{N-1} A t_{j}, ; \quad A_{\text {vazio }}=\sum_{k=1}^{M-1} A t_{k},
$$

sendo $A_{\text {ext }}$ a área compreendida dentro do contorno externo da envoltória e $A_{\text {vazio }}$ a área do vazio. As áreas são calculadas como o somatório das áreas dos trapézios $\left(A_{t}\right)$ determinadas por

$$
A t_{i}=\frac{[r(i+1)+r(i)][z(i+1)-z(i)]}{2} .
$$

O baricentro é calculado como:

$$
r_{G}=\frac{\sum_{j=1}^{N-1}\left(r_{G} t_{j} A t_{j}\right)-\sum_{k=1}^{M-1}\left(r_{G} t_{k} A t_{k}\right)}{A},
$$

sendo

$$
r_{G} t_{i}=\frac{r(i)^{2}+r(i+1)^{2}+r(i) r(i+1)}{3[r(i)+r(i+1)]} .
$$




\section{Revisão sobre algoritmos genéticos}

Algoritmo genético é um processo pseudo-randômico de otimização que utiliza valores aleatórios a serem atribuídos às várias variáveis que compõem o sistema. Denomina-se por indivíduo o conjunto de valores atribuídos para cada variável (Kirkpatrick [7]). A probabilidade ou aptidão de cada indivíduo é um valor que representa o grau de adaptação deste, ou seja, o quão próximo este conjunto de valores está do conjunto solução do problema, em relação a um agrupamento de $n$ indivíduos.

A programação genética faz com que sejam produzidas várias gerações de indivíduos com o mesmo número de indivíduos da geração inicial. A definição dos indivíduos das gerações seguintes é baseada em três operadores principais: seleção, cruzamento e mutação.

No processo de seleção, os indivíduos de baixa aptidão são eliminados e os de aptidão mais alta são repetidos com uma taxa de repetição proporcional à respectiva aptidão.

O cruzamento é geralmente feito após a seleção e consiste na criação de indivíduos que contém características genéticas de outros dois indivíduos genitores. Alguns algoritmos genéticos são baseados no cruzamento heurístico, para o qual o indivíduo descendente tende mais para o indivíduo genitor de que apresenta melhor valor para a função objetivo, similarmente ao conceito de dominância da biologia. A probabilidade de ocorrência do cruzamento entre dois indivíduos pode ser determinada pelo usuário, sendo $25 \%$ um valor bastante utilizado.

O processo de mutação tem um papel importante e necessário, porque a reprodução e o cruzamento podem perder material genético potencialmente útil. O operador de mutação protege os algoritmos genéticos contra perdas irreparáveis. Tomada isoladamente, a mutação se constituiria na exploração aleatória do espaço das cadeias. Utilizada com cuidado, juntamente com os outros dois operadores, protege-se o procedimento da perda prematura de informações importantes. A probabilidade de ocorrência da mutação mais utilizada é de 1\%. Aplicados estes três operadores, gera-se uma nova população de indivíduos, ou seja, tem-se uma nova geração.

\section{Formulação do problema de otimização}

Seja o problema de otimização definido da seguinte forma:

$$
\max F(x)=V
$$

sendo

$$
x=\left[\begin{array}{lllllll}
a_{1} & a_{2} & a_{3} & d_{2} & d_{3} & \alpha_{1} & \alpha_{2}
\end{array}\right] ; \quad x_{\mathrm{inf}} \leq x_{i} \leq x_{\mathrm{sup}}
$$

sujeito à

$$
\left\{\begin{array}{l}
g_{1}(x)=\frac{\partial^{2} f}{\partial \theta_{3}^{2}} \neq 0 \\
g_{2}(x)=z-z_{\max } \leq 0 \\
g_{3}(x)=z_{\min }-z \leq 0
\end{array}\right.
$$


Utilizou-se um programa elaborado em MATLAB para o cálculo do volume $V$ do espaço de trabalho, equação (3.5), em conjunto com o programa de otimização GAOT. As variáveis de otimização são os parâmetros $a_{1}, d_{2}, a_{2}, d_{3}, a_{3}, \alpha_{1}, \alpha_{2}$, limitados inferiormente e superiormente pelos vetores $x_{\text {inf }}$ e $x_{\text {sup }}$. Cada indivíduo representa uma configuração diferente do robô e o projeto ótimo corresponde ao de maior volume do espaço de trabalho.

A primeira restrição, $g_{1}(x)$, está relacionada com a regularidade da envoltória. Esta será irregular se houver a presença de pontos de singularidade, que podem ser detectados pela anulação do valor da segunda derivada da função $f$ em relação à $\theta_{3}$, conforme estudado em Bergamaschi et al [3]. A segunda e a terceira restrição, $g_{2}(x)$ e $g_{3}(x)$, referem-se aos limites dimensionais da envoltória em relação ao eixo- $z$.

Quando alguma destas restrições não é obedecida, impõe-se um valor reduzido à função objetivo, eliminando-se, assim, o indivíduo correspondente durante o processo de seleção do algoritmo.

\section{Aplicações Numéricas}

A seguir, serão apresentados alguns exemplos para a comprovação da metodologia desenvolvida.

Exemplo 6.1. 3000 indivíduos em uma geração.

$$
\begin{gathered}
x_{\mathrm{inf}}=\left[\begin{array}{ccccccc}
0 & 0 & 0 & 0 & 0 & 0 & 0
\end{array}\right] \\
x_{\mathrm{sup}}=\left[\begin{array}{ccccccc}
1 & 1 & 1 & 1 & 1 & \frac{\pi}{2} & \frac{\pi}{2}
\end{array}\right] \\
z_{\min }=0 \leq z \leq z_{\max }=10 .
\end{gathered}
$$

Para uma única geração, obteve-se um volume final de 10,2246 unidades de volume (u.v.) e a envoltória está mostrada na Figura 3-a. A Figura 3-b representa o gráfico da restrição $g_{1}(x)$ para cada contorno da envoltória (interno e externo).

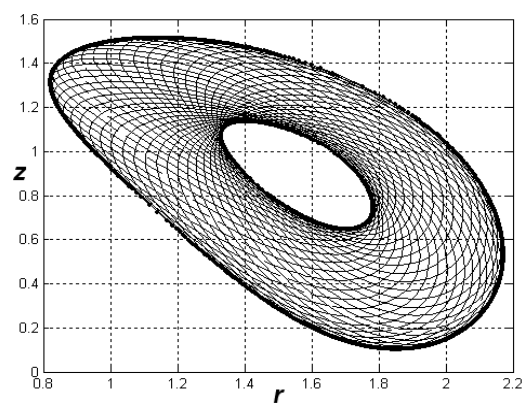

(a)

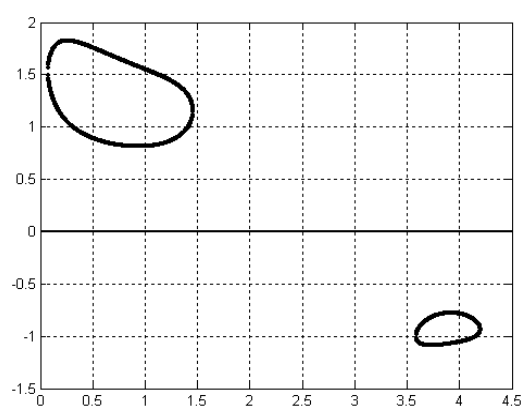

(b)

Figura 3: (a) Seção radial, (b) restrição $g_{1}(x)$ resultante para o Exemplo 6.1.

Os parâmetros ótimos obtidos para o manipulador para este exemplo são:

$$
x=\left[\begin{array}{lllllll}
0,9232 & 0,2096 & 0,5857 & 0,5475 & 0,9267 & 0,9499 & 0.1952
\end{array}\right] .
$$


Exemplo 6.2. 3000 indivíduos em 1000 gerações (com restrições idênticas às do Exemplo 6.1).

Obteve-se como resultado um volume final de 50,78 u.v. A envoltória está mostrada na Figura 4-a e a restrição $g_{1}(x)$ na Figura 4-b. Os parâmetros ótimos obtidos para o manipulador para este exemplo são:

$$
x=\left[\begin{array}{lllllll}
1,0000 & 0,8888 & 0,8238 & 0,9804 & 1,0000 & 0,7445 & 0,3497
\end{array}\right]
$$

Verifica-se através destes gráficos que não houve formação de pontos de singularidades nas curvas da envoltória, ou seja, a restrição $g_{1}$ foi obedecida. As restrições $g_{2}$ e $g_{3}$ também foram obedecidas.

No eixo- $z$, os pontos estão dentro dos limites impostos. Verifica-se também a redução da área vazia, que ocorreu durante o processo de otimização.

Comparando os exemplos 6.1 e 6.2, verifica-se que, após 1000 gerações, o espaço de trabalho apresentou um acréscimo de 496,64\% em seu volume, em relação à primeira geração, para a qual o projeto ótimo é escolhido apenas por uma seleção entre os indivíduos definidos aleatoriamente.

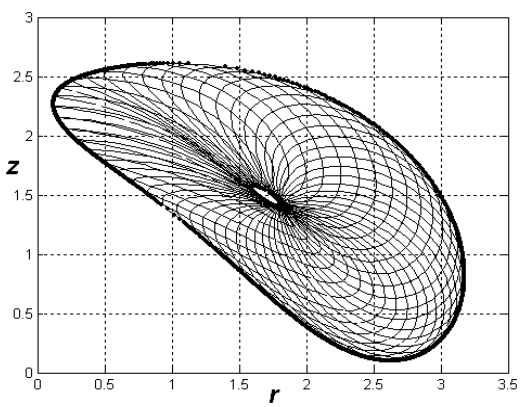

(a)

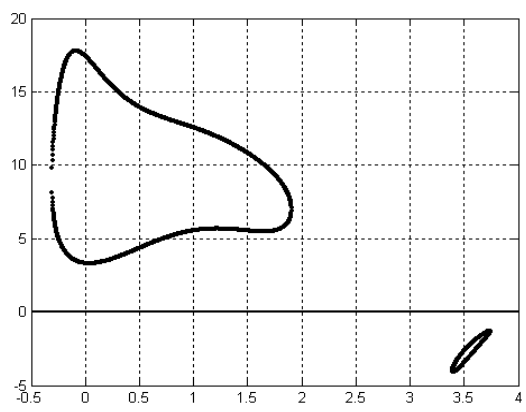

(b)

Figura 4: (a) Seção radial, (b) restrição $g_{1}(x)$ resultante para o Exemplo 6.2.

\section{Conclusões}

Algoritmos genéticos foram aplicados na solução do projeto ótimo de um manipulador 3R, formulado como um problema de otimização cujo objetivo é maximizar o volume do espaço de trabalho. A restrição imposta de que apenas envoltórias regulares sejam aceitas foi obedecida. Além disso, outras restrições foram impostas ao problema para delimitar os limites desejados da região a ser alcançada e obter as dimensões do robô de acordo com valores práticos. Os exemplos numéricos comprovam que o problema de maximização foi solucionado e que é possível a obtenção de bons resultados através da metodologia apresentada.

A possibilidade de utilização de métodos híbridos de otimização, onde os algoritmos genéticos serão aplicados em conjunto com técnicas seqüências e a utilização de funções de penalidade para representação das restrições são as propostas dos autores para futuros estudos neste assunto. 


\title{
8. Agradecimentos
}

Os autores agradecem à Fundação de Amparo à Pesquisa de Minas Gerais (Fapemig) pelo apoio financeiro a este trabalho (número do processo: TEC 084/02).

\begin{abstract}
The objective of this work is the optimal design 3R robot manipulators, considering only the workspace in which envelope is regular. The optimization is characterized by obtaining a workspace with maximum volume and minimum robot dimensions. The study of the envelope is important for the development of the manipulator's design, because it represents the boundary of its workspace. Its form depends on the architecture and on the robot's dimensions. Those parameters should be determined to obtain the maximum volume. The investigated workspace can usually present voids, and its envelope can present singularities that are called cusp points. The envelope is classified as irregular when these singularities appear and they are not considered during the optimization process. Optimization was performed by using genetic algorithms. The authors have developed a computational code to calculate the mathematical model. Some numeric examples are presented to illustrate the proposed methodology.
\end{abstract}

\section{Referências}

[1] K. Abdel-Malek, H-J Yeh e S. Othman, Understanding voids in the workspace of serial robot manipulators, em "Proceedings of $23^{\text {rd }}$ ASME Design Engineering Technical Conf.", Baltimore, Maryland, 2000.

[2] F.P. Beer e E.R. Johnston, Jr., "Mecânica Vetorial Para Engenheiros", 5. ed., Vol. 1 e 2, São Paulo: Makron, McGraw-Hill, Brasil, 1991.

[3] P.R. Bergamaschi, A.C. Nogueira e S.F.P. Saramago, An optimal design of 3R manipulators taking into account regular workspace boundary, em "Proceed. of XVII Cong. Bras. de Eng. Mecânica (COBEM)", ABCM, 2003.

[4] J.W. Bruce e P.J. Giblin, "Curves and Singularities: a geometrical introduction to singularity theory", Cambridge University Press, Great Britain, 2nd. Edition, 1992.

[5] M. Ceccarelli, A formulation for the workspace boundary of general N-revolute manipulators, Mechanism and Machine Theory, 31 (1996), 637-646.

[6] C.R. Houck, J.A. Joines e M.G. Kay, "A Genetic Algorithm for Function Optimization: a Matlab Implementation", NCSU-IE Technical Report,1995.

[7] S. Kirkpatrick, C.D. Gelatt Jr. e M.P. Vecchi, Optimization by simulated annealing, Science, 220, No. 4598, (1983), 671-680.

[8] A. Kumar e K.J. Waldron, The workspaces of a mechanical manipulator, ASME Jnl of Mechanical Design, 103 (1981), 665-672. 
[9] C. Lanni, S.F.P. Saramago e M. Ceccarelli,Optimum design of general 3R manipulators by using traditional and random search optimization techniques and simulated annealing, Revista Brasileira de Ciências Mecânicas, 24, No. 4 (2002), 293-301.

[10] R.P. Paul, "Robot Manipulators: Mathematics, Programming, and Control", Massachusetts Institute of Technology, USA, 1981.

[11] B. Roth, Performance evaluation of manipulators from a kinematic viewpoint, "Special Publication, Performance Evaluation of Programmable Robots and Manipulators", (1975), 39-61.

[12] S.F.P. Saramago, E. Ottaviano e M. Ceccarelli, A Characterization of the Workspace Boundary of Three-Revolute Manipulators, em "Proc. of Design Eng. Technical Conferences (DETC'02)", ASME, Montreal, 34342-34352, 2002.

[13] P. Wenger, Some Guidelines for the Kinematic Design of New Manipulators, Mechanism and Machine Theory, 35 (2000), 437-449. 